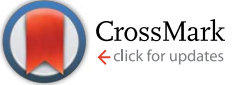

Cite this: RSC Adv., 2017, 7, 10939

Received 30th November 2016 Accepted 30th January 2017

DOI: $10.1039 / c 6 r a 27542 g$

rsc.li/rsc-advances

\section{Antifungal activity and mechanism of heat- transformed ginsenosides from notoginseng against Epidermophyton floccosum, Trichophyton rubrum, and Trichophyton mentagrophytes}

\begin{abstract}
Peng Xue, $\dagger^{\mathrm{ab}}$ Xiushi Yang, $\uparrow^{\mathrm{a}}$ Xiaoyan Sun ${ }^{\mathrm{ab}}$ and Guixing Ren*ab
Notoginseng, the root of Panax notoginseng, has been used to treat wounds and ulcers for several years in China. However, a rare report was found on the antifungal effect of saponin from notoginseng or a heattransformed one, particularly against dermatophytes. In this study, notoginseng saponin (NS) was heated in a reaction kettle to produce heat-transformed ginsenoside (HTS). Chromatography and spectroscopic characterization revealed that five polar ginsenosides in NS (notoginsenoside-R1, ginsenoside-Rg1, -Re, -Rb2, -Rd) were almost transformed to three less polar ginsenosides in HTS (ginsenoside-Rk3, -Rh4, -Rh5). HTS showed higher inhibitory activity than that of NS against Epidermophyton floccosum, Trichophyton rubrum, and Trichophyton mentagrophytes, with the lower minimum inhibitory concentration (MIC, 16 or $31.25 \mu \mathrm{g} \mathrm{mL}^{-1}$ ) and minimum fungicidal concentration (MFC, 125 or $250 \mu \mathrm{g}$ $\mathrm{mL}^{-1}$ ). Moreover, less polar ginsenosides-Rk3, -Rh4, and -Rh5 showed the highest inhibitory activity among the ginsenoside monomers with the lowest MIC $\left(16 \mu \mathrm{g} \mathrm{mL}^{-1}\right)$ and MFC $\left(62.5 \mu \mathrm{g} \mathrm{mL} \mathrm{m}^{-1}\right)$. It was also found that the antifungal activity of individual ginsenosides was negatively correlated with the number of their sugar moieties. Moreover, the antifungal activity of HTS might be attributed to the damage of cell membrane and decrease of membrane potential, easily caused by less polar ginsenosides. Thus, the results indicated that HTS could be applied as antifungal agents to treat ringworm.
\end{abstract}

\section{Introduction}

Dermatophytes are a group of fungi that induce dermatophytosis (commonly known as ringworm or tinea) of human keratinous tissues such as skin, hair, and nails. ${ }^{1}$ According to the data of the World Health Organization, dermatophytes affected about $25 \%$ of the world population with dermatophytosis. ${ }^{2}$ Among the fungal species isolated from infected skins, Epidermophyton floccosum (E. floccosum), Trichophyton rubrum (T. rubrum), and Trichophyton mentagrophytes (T. mentagrophytes) were the three frequent dermatophytes in clinical cases of tinea pedis, tinea unguium, tinea corporis, and tinea cruris. ${ }^{3}$ These fungi were also reported to induce dermatophytes in clinical cases, ${ }^{4,5}$ and they were chosen to evaluate the antifungal effect of a drug to cure infected skins. ${ }^{6-9}$ Allylamine and azole have been used to treat dermatophytosis for a long time, but they exhibit several side effects, such as cardiac toxicity, hepatotoxicity, inhibited bone marrow, cardiovascular toxicity,

${ }^{a}$ Institute of Crop Sciences, Chinese Academy of Agricultural Sciences, Beijing, 100081, People's Republic of China. E-mail: renguixing@caas.cn; Fax: +86-10-6215-6596; Tel: +86-10-6211-5596

${ }^{b}$ College of Food Science and Technology, Qilu University of Technology, Jinan, 250353, People's Republic of China

$\dagger$ The first two authors contributed equally to this work. and vascular toxicity. ${ }^{10-15}$ In order to find an alternative treatment, efforts have been made on several phytochemicals with the activity against fungal pathogens, such as the essential oil from aerial parts of Artemisia sieberi Besser, Perilla frutescens (L.) Britt., and the flowers of Lavandula stoechas L., Thymus herbabarona Loisel. ${ }^{16-18}$

Notoginseng, the root of Panax notoginseng (Burkill) F. H. Chen (Araliaceae), has been used for thousands of years as food and herbal medicine in China. It was also included as a dietary supplement by the US Dietary Supplement Health and Education Act in $1994 .{ }^{19}$ Saponin was reported as the major active ingredient in notoginseng and more than 70 saponins were isolated from this medicinal plant. These saponins showed haemostatic, antioxidant, and hypolipidemic effects. ${ }^{20}$ Saponins from natural plants, such as minutoside $\mathrm{B}$, minutoside $\mathrm{C}$, 3-O- $\alpha$-L-arabinopyranosyl hederagenin 28-O- $\alpha$-L-rhamnopyranosylester, disporoside $\mathrm{A}$, and

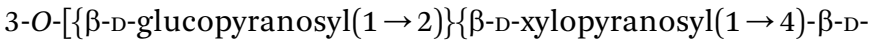
glucopyranosyl]-(25S)-5 $\beta$-spirostan-3 $\beta$-ol, exhibited strong antifungal activity. ${ }^{21-24}$ The polarity of saponin (or ginsenosides in notoginseng) with more than two glucose molecules was higher than the ginsenosides containing only one or two glucose molecules. Ginsenoside with high polarity could be easily dissociated using the reversed-phase separation. ${ }^{25}$ Less polar ginsenosides could be isolated from natural notoginseng, but the yield was very low. 
Heat transformation was successfully applied to produce more effective chemicals from several natural plants, such as Panax ginseng, Panax quinquefolius, and Panax notoginseng with a high yield. ${ }^{26-28}$ However, a rare report was found on the antifungal effect of heat-transformed ginsenosides from notoginseng, particularly against dermatophytes. In this sense, in this study, notoginseng was heat-transformed to get less polar ginsenosides, and the antifungal activity and related mechanism of action were also investigated.

\section{Materials and methods}

\subsection{Materials}

High performance liquid chromatography (HPLC) grade acetonitrile and methanol were obtained from Merck (Darmstadt, Germany). Deionized water $\left(18 \mathrm{MV} \mathrm{cm}^{-1}\right)$ was prepared by passing distilled water through a Milli-Q system (Millipore, Bedford, MA, USA). The raw notoginseng saponins (NS) were purchased from Yunnan Kingtide Notoginseng Industry Co., Ltd. (Kunming, China). Reference ginsenosides of -R1, -Re, -Rg1, -Rb1, and -Rd, with $>98 \%$ purity were purchased from the National Institute for the Control of Pharmaceutical and Biological Products (Beijing, China). Ginsenoside-Rk3, -Rh4, and -Rh5, (>95\% purity) were isolated in our laboratory and further elucidated by ${ }^{1} \mathrm{H}-,{ }^{13} \mathrm{C}-\mathrm{NMR}$, and MS. Miconazole nitrate and dimethyl sulfoxide (DMSO) were purchased from J \& $\mathrm{K}$ (Shanghai, China). Sabouraud agar modified (SAM) was purchased from Beijing Solarbio Biotech Co., Ltd. (Beijing, China). The other chemicals were of analytical or HPLC grade.

\subsection{Preparation of less polar ginsenosides}

The preparation of less polar ginsenoside was performed according to the previous report with slight modification. ${ }^{29}$ NS was heated in an MLS-3750 autoclave (Sanyo, Osaka, Japan) at $130{ }^{\circ} \mathrm{C}$ for $3 \mathrm{~h}$ to obtain heat-transformed saponins (HTS). HTS was loaded onto a LH-20 column and sequentially eluted with water and 95\% methanol about three BV, respectively. The fractions were collected and evaporated using a rotary evaporator (Buchi, Flawil, St. Gallen, Switzerland) at $55{ }^{\circ} \mathrm{C}$ to remove methanol and then lyophilized to obtain the dry powder. These fractions were subjected to HPLC analysis.

\subsection{HPLC analysis}

The analysis of less polar ginsenoside was performed according to the previous report with slight modification. ${ }^{29}$ Chromatographic analysis for the ginsenosides was performed using a SHIMADZU Prominence LC-20A HPLC instrument (Shimadzu Corporation, Kyoto, Japan) equipped with a YMC-Pack ODS-AM column (4.6 mm $\times 250 \mathrm{~mm}$, YMC Co., Ltd., Kyoto, Japan). The detection wavelength was set at $202 \mathrm{~nm}$. The mobile phase consisted of water (A) and acetonitrile (B). A gradient elution was used as follows: $25 \% \mathrm{~B}$ at $0-5 \mathrm{~min}, 30-32 \% \mathrm{~B}$ at $5-14 \mathrm{~min}$, $32-38 \% \mathrm{~B}$ at $14-28 \mathrm{~min}, 38-46 \% \mathrm{~B}$ at $28-30 \mathrm{~min}, 46-74 \% \mathrm{~B}$ at $30-50 \mathrm{~min}, 74-80 \% \mathrm{~B}$ at $50-51 \mathrm{~min}, 80-90 \% \mathrm{~B}$ at $51-60 \mathrm{~min}, 90-$ $100 \% \mathrm{~B}$ at $60-65 \mathrm{~min}, 100-25 \% \mathrm{~B}$ at $65-70 \mathrm{~min}$. The flow rate

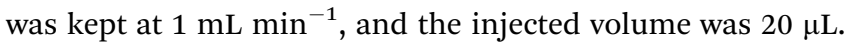

\subsection{HPLC preparation}

An Agilent 1200 HPLC system (Agilent Technologies, Santa Clara, CA, USA) was used to prepare ginsenosides-Rk3, -Rh4, and -Rh5. The chromatographic separation was achieved on a Grace Platinum EPS-C18 preparative column $(22 \mathrm{~mm} \times 250$ $\mathrm{mm}, 5 \mu \mathrm{m}$ ) with a solvent flow rate of $10 \mathrm{~mL} \mathrm{~min}^{-1}$ at room temperature. HPLC preparation of less ginsenosides was performed according to the analytical method. ${ }^{29}$ The mobile phase was composed of water (A) and acetonitrile (B). The collected samples were evaporated under vacuum, lyophilized, and then stored at $4{ }^{\circ} \mathrm{C}$.

\subsection{Antifungal assays}

All standard fungal strains were purchased from Guangdong Microbiology Culture Center (Guangzhou, China). Epidermophyton floccosum (ATCC 52066), Trichophyton rubrum (ATCC 28188), and Trichophyton mentagrophytes (ATCC 9533) were cultured in SAM for $72 \mathrm{~h}$ at $38{ }^{\circ} \mathrm{C}$ in a YQX-II Anaerobic incubator (Shanghai, China) for further use. The antifungal experiment was performed using the micro dilution method of Yang and Jiang. ${ }^{30}$ Cell suspensions were diluted in sterile SAM to provide initial cell counts of about $10^{8}$ colony-forming units per $\mathrm{mL}\left(\mathrm{CFU} \mathrm{mL} \mathrm{m}^{-1}\right)$. Miconazole nitrate $(2 \mathrm{mg}$ ) was dissolved in DMSO $(2 \mathrm{~mL})$ for the subsequent antifungal experiments as the positive control. All samples were dissolved in DMSO at the concentration of $10 \mathrm{mg} \mathrm{mL}^{-1}$.

2.5.1 Minimum inhibitory concentration and minimum fungicidal concentration. The minimum inhibitory concentration (MIC) and minimum fungicidal concentration (MFC) were determined using the micro dilution method of Kubo and Himejima. ${ }^{31}$ Samples were added to the SAM medium to prepare serial 2-fold dilutions (from 1 to $0.125 \mathrm{mg} \mathrm{mL}^{-1}$ for NS and HTS, from 1 to $0.016 \mathrm{mg} \mathrm{mL}^{-1}$ for each ginsenoside monomer). Each dilution $(100 \mu \mathrm{L})$ and fungal liquid cultured media $(100 \mu \mathrm{L})$ were added to 96 -well microplates, with sterile SAM medium as a control. After inoculation, the microplates were incubated at $38{ }^{\circ} \mathrm{C}$. MIC corresponded to the lowest concentration of each tested compound that resulted in no visible mycelial growth after 3 days of incubation. MFC corresponded to the lowest concentration of each tested compound (including negative and positive controls), in which no recovery of a microorganism was observed after 7 days. Broths with $6.4 \%$ $(\mathrm{v} / \mathrm{v})$ DMSO were set for growth of control, and miconazole nitrate was used for positive control. Each experiment was performed in triplicate.

2.5.2 Integrity of cell membrane. According to the method of Zhang, ${ }^{32}$ the integrity of the cell membrane was evaluated by measuring the release of cell constituents into cell suspension. Cells from the suspension ( $5 \mathrm{~mL}$ ) of E. floccosum, T. rubrum and T. mentagrophytes were collected by centrifugation for $3 \mathrm{~min}$ at $6000 \times g$. Then, they were washed three times thoroughly and re-suspended in phosphate-buffered saline (PBS, 0.1 M, pH 7.4). The $5 \mathrm{~mL}$ washed suspension was incubated at $38^{\circ} \mathrm{C}$ for $28 \mathrm{~h}$ in the presence of different concentrations of HTS (MIC and MFC), NS (0.125 $\mathrm{mg} \mathrm{mL}^{-1}$ ) and DMSO. Then, suspensions were centrifuged at $6500 \times g$ for $3 \mathrm{~min}$. After that, supernatants were 
collected, diluted with PBS, and transferred into a 96-well microplate. The absorption at $260 \mathrm{~nm}$ was measured using a UV-Vis spectrophotometer (Molecular Devices, Sunnyvale, CA, USA). Since the saponin samples showed a little yellow color, their absorptions were corrected using the suspension of PBS (0.1 M, pH 7.4) containing the same concentration of each sample after $2 \mathrm{~min}$ reaction with the tested strains. The untreated cells (control) were corrected with DMSO (6.4\%). Results were expressed in terms of optical density of $260 \mathrm{~nm}$ absorbing materials in each interval with respect to the last time. In addition, suspension was also collected to determine the concentration of protein according to Bradford's method. ${ }^{33}$ Each experiment was performed in triplicate.

2.5.3 Membrane potential. To analyze the effects of HTS on the metabolic activity changes of fungi, the membrane potential (MP) of the fungi was measured according to the rhodamine fluorescence method as described by Comas and Vives-Rego with some modifications. ${ }^{34}$ Fungal cells were incubated in SAM medium at $38{ }^{\circ} \mathrm{C}$ for $24 \mathrm{~h}$. The cell solutions $\left(1 \times 10^{7} \mathrm{CFU}\right.$ $\mathrm{mL}^{-1}$ ) were added with different concentrations of HTS (MIC and MBC levels) and NS $\left(0.125 \mathrm{mg} \mathrm{mL}^{-1}\right)$ and then incubated for $3 \mathrm{~h}$; the untreated cells (control) were corrected with DMSO (6.4\%). Rhodamine 123 was added to PBS to obtain a $1 \mathrm{mg} \mathrm{mL}$ stock solution. The suspensions were washed with PBS. Rhodamine 123 was then added to obtain a final concentration of $2 \mu \mathrm{g} \mathrm{mL}{ }^{-1}$. After standing in dark for $30 \mathrm{~min}$, samples were completely washed and resuspended in PBS. The cell suspension was transferred into a 96-well microplate and then placed in the spectrofluorometer (F-4500, HITACHI, Japan). Rhodamine 123 fluorescence was excited at $480 \mathrm{~nm}$ and emission wavelength was collected at $530 \mathrm{~nm}$. The data was expressed by mean fluorescence intensity (MFI). Each experiment was performed in triplicate.

\subsection{Statistical analysis}

Data were presented as the mean of three replicates \pm standard deviation. Statistical analysis of the results was done with
Microsoft Excel 2007 and SPSS software for windows (version 13.0) using a computer (Lenovo, Yangtian B 41) equipped with Windows 7 operating system software. One-way ANOVA with Duncan's multiple range test was used to analyze the results. A $p$-value of $<0.05$ was used to determine significant differences between groups.

\section{Results and discussion}

\subsection{Characterization of ginsenosides}

Seven ginsenosides were simultaneously identified according to the retention time, HPLC-ESI-MS ion fragments and NMR. The results of ion fragments of ginsenosides were consistent with a previous report. ${ }^{35}$ The typical HPLC-UV chromatograms of ginsenosides in raw and transformed fractions are shown in Fig. 1. Peaks (peak 2 contains ginsenoside-Rg1 and -Re) were separated under gradient elution. Contents of ginsenoside monomers in NS and HTS were shown in Table 1. It was found that the reference compounds showed good linearity $\left(R^{2} \geq\right.$ 0.996), except ginsenoside-Rh4 $\left(R^{2}=0.9911\right)$. The average recovery of the ginsenosides ranged from $98.7 \%$ to $103.1 \%$ with relative standard deviation (RSD) less than $3.0 \%$.

NS contained five types of polar ginsenosides $(0.791 \pm$ $\left.0.003 \mathrm{mg} \mathrm{mg}^{-1}\right)$, including -R1 (0.164 $\left.\pm 0.003 \mathrm{mg} \mathrm{mg}^{-1}\right),-\mathrm{Re} /$ Rg1 $\left(0.285 \pm 0.004 \mathrm{mg} \mathrm{mg}^{-1}\right),-\mathrm{Rb} 1\left(0.272 \pm 0.003 \mathrm{mg} \mathrm{mg}^{-1}\right)$ and $-\mathrm{Rd}\left(0.07 \pm 0.001 \mathrm{mg} \mathrm{mg}^{-1}\right)$. However, after heat treatment, these ginsenosides were transformed into the less polar ginsenosides of -Rk3, -Rh4, and -Rh5 in HTS. ${ }^{36}$ The content of total saponins in HTS $\left(0.707 \pm 0.002 \mathrm{mg} \mathrm{mg}^{-1}\right)$ was slightly lower than that of NS.

\subsection{Antifungal activities of NS and HTS}

The MIC and MFC of NS and HTS against the three dermatophytes are presented in Table 2 . NS showed an inhibitory effect on the three fungi with MIC of $250 \mu \mathrm{g} \mathrm{mL}{ }^{-1}$. At the concentration of $1000 \mu \mathrm{g} \mathrm{mL} \mathrm{m}^{-1}$, NS showed a fungicidal effect on $T$. rubrum and T. mentagrophytes. However, HTS exhibited stronger
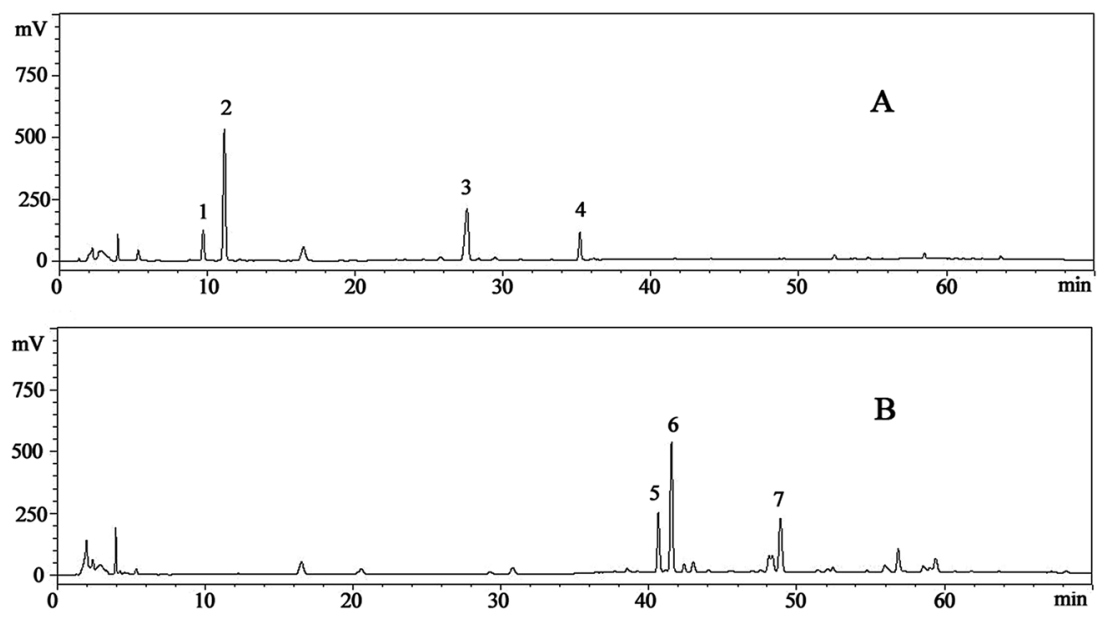

Fig. 1 HPLC chromatogram of ginsenosides in NS (A) and HTS (B). NS, raw notoginseng saponins; HTS, heat-transformed saponins. Identification of peaks: 1, R1; 2, Rg1/Re; 3, Rb1; 4, Rd; 5, Rk3; 6, Rh4; 7, Rh5. 
Table 1 Ginsenosides contents in NS and $\mathrm{HTS}^{a}$

\begin{tabular}{|c|c|c|c|c|c|}
\hline Ginsenoside & Retention time & Calibration curve & $R^{2}$ & $\mathrm{NS}\left(\mathrm{mg} \mathrm{mg}{ }^{-1}\right)$ & $\operatorname{HTS}\left(\mathrm{mg} \mathrm{mg}{ }^{-1}\right)$ \\
\hline $\mathrm{R} 1$ & 9.718 & $y=356751 x-1000000$ & 0.9973 & $0.164 \pm 0.003$ & n.d \\
\hline $\operatorname{Rg} 1 / \operatorname{Re}$ & 11.158 & $y=481999 x+93235$ & 0.9999 & $0.285 \pm 0.004$ & n.d \\
\hline $\mathrm{Rd}$ & 35.201 & $y=271969 x+427367$ & 0.9961 & $0.070 \pm 0.001$ & n.d \\
\hline Rk3 & 40.647 & $y=831162 x+913532$ & 0.9865 & n.d & $0.249 \pm 0.002$ \\
\hline Rh4 & 41.526 & $y=1000000+691802$ & 0.9911 & n.d & $0.355 \pm 0.001$ \\
\hline
\end{tabular}

Table 2 MIC and MFC of NS and HTS against the dermatophytes $\left(\mu \mathrm{g} \mathrm{mL}^{-1}\right)^{a}$

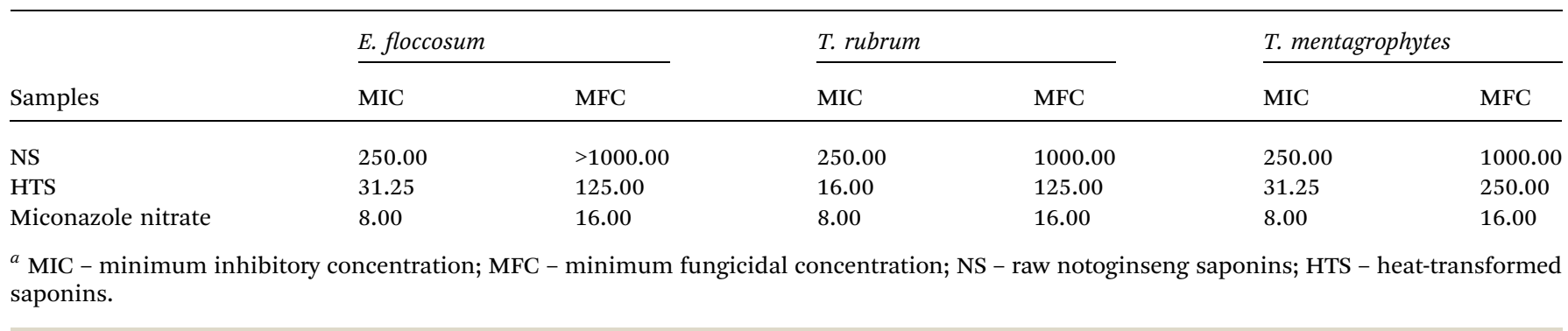

antifungal activity than that of NS with lower MIC and MFC. Among the three fungi, T. rubrum was the most susceptible to HTS, with the lowest MIC. The MIC of HTS against T. rubrum was just double than that of miconazole nitrate, which implied that HTS could also be used as a potent antifungal agent. According to previous investigations, ${ }^{37,38}$ ginseng extracts containing polar ginsenosides-Rb1 and - Rg1 did not affect the growth rate of $P$. aeruginosa, $S$. aureus, or $H$. pylori. Interestingly, less polar ginsenosides produced from American ginseng by heat transformation showed inhibitory activity against antiPropionibacterium acnes. ${ }^{39}$ In addition, less polar ginsenosides in heat-transformed saponins from American ginseng leaf-stem also showed a significant anti-bacterial effect. ${ }^{29}$ It might be concluded that the antimicrobial activity of ginsenosides was related to their polarity and less polar ginsenoside was more effective than the high polar one against fungi.. ${ }^{29}$

\subsection{Antifungal activities of different ginsenoside monomers and structure-activity relationship analysis}

In order to find which ginsenoside monomers were responsible for the antifungal effects of NS and HTS, all 8 ginsenoside monomers were prepared and their antifungal activities were tested. As shown in Table 3, it was found that -Rg1 exhibited the lowest MIC and MFC among the five ginsenosides in NS. Referring to HTS, the three ginsenoside monomers of -Rk3, -Rh4, and -Rh5 displayed the same role, being similar in both MIC and MFC. The three less polar ginsenosides showed superior antifungal effect than that of the other five polar monomers.

As shown in Fig. 2 and Table 3, ginsenosides-R1, -Re, -Rb1 and -Rd, with more than three sugar moieties, showed
Table 3 MIC and MFC of different ginsenoside monomers against the dermatophytes $\left(\mu \mathrm{g} \mathrm{mL}^{-1}\right)^{a}$

\begin{tabular}{|c|c|c|c|c|c|c|}
\hline \multirow[b]{2}{*}{ Samples } & \multicolumn{2}{|c|}{ E. floccosum } & \multicolumn{2}{|c|}{ T. rubrum } & \multicolumn{2}{|c|}{$\begin{array}{l}\text { T. } \\
\text { mentagrophytes }\end{array}$} \\
\hline & MIC & MFC & MIC & MFC & MIC & MFC \\
\hline R1 & 250 & $>500$ & 250 & $>500$ & 250 & $>500$ \\
\hline Rg1 & 125 & 250 & 62.5 & 250 & 125 & 250 \\
\hline $\mathrm{Re}$ & 250 & $>500$ & 250 & $>500$ & 250 & $>500$ \\
\hline $\mathrm{Rb} 1$ & 250 & $>500$ & 250 & $>500$ & 250 & $>500$ \\
\hline $\mathrm{Rd}$ & 250 & $>500$ & 250 & $>500$ & 250 & $>500$ \\
\hline Rk3 & 16 & 62.5 & 16 & 62.5 & 16 & 62.5 \\
\hline $\mathrm{Rh} 4$ & 16 & 62.5 & 16 & 62.5 & 16 & 62.5 \\
\hline Rh5 & 16 & 62.5 & 16 & 62.5 & 16 & 62.5 \\
\hline
\end{tabular}

${ }^{a}$ MIC - minimum inhibitory concentration; MFC - minimum fungicidal concentration; NS - raw notoginseng saponins; HTS - heattransformed saponins.

inhibitory effects on fungi with an MIC of $250 \mu \mathrm{g} \mathrm{mL}^{-1}$. Ginsenoside-Rg1, with two sugar chains, showed a higher antifungal effect than that of the former ones with the lower MIC values. Ginsenosides-Rk3, -Rh4, and -Rh5, with one sugar chain, showed the highest inhibitory activity with the lowest MIC values among the monomers. It might be concluded that the antifungal effect of ginsenosides was negatively correlated with the number of sugar moieties.

\subsection{Antifungal mechanism}

The integrity of the cytoplasmic membrane is a critical factor to fungus growth, metabolism, and reproduction. Analyzing the 

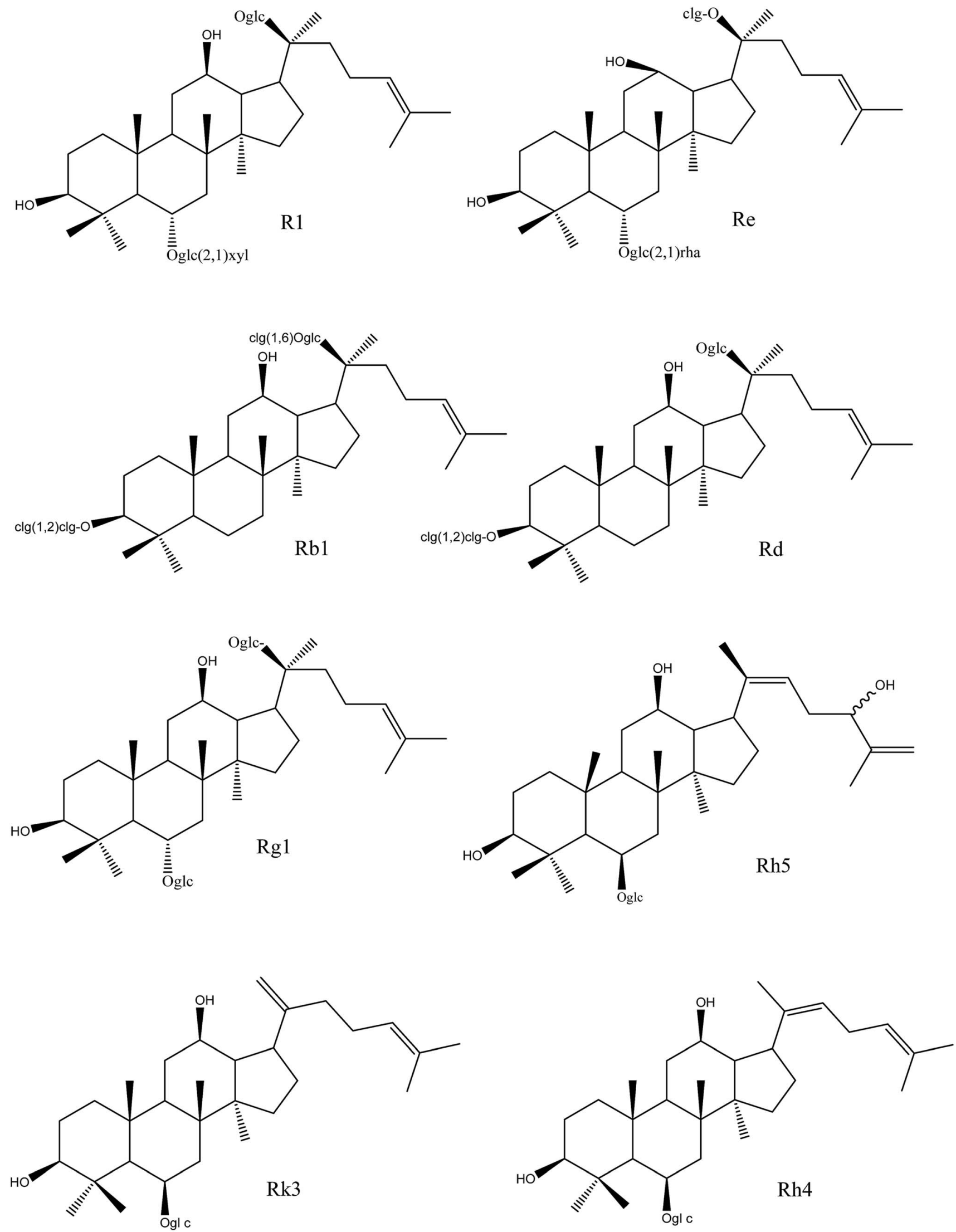

Fig. 2 Chemical structures of different ginsenoside monomers in NS and HTS. NS, raw notoginseng saponins; HTS, heat-transformed saponins. 
leakage of cell constituents can therefore provide further insight into the mechanism of antifungal action. Investigation on the release of fungal cell constituents, such as DNA and protein, provides interesting information about the integrity of the cell membrane. ${ }^{40}$ The results of $\mathrm{OD}_{260 \mathrm{~nm}}$ and protein concentrations are shown in Fig. 3 and 4, respectively. With the presence of HTS, the $\mathrm{OD}_{260} \mathrm{~nm}$ values for all the three
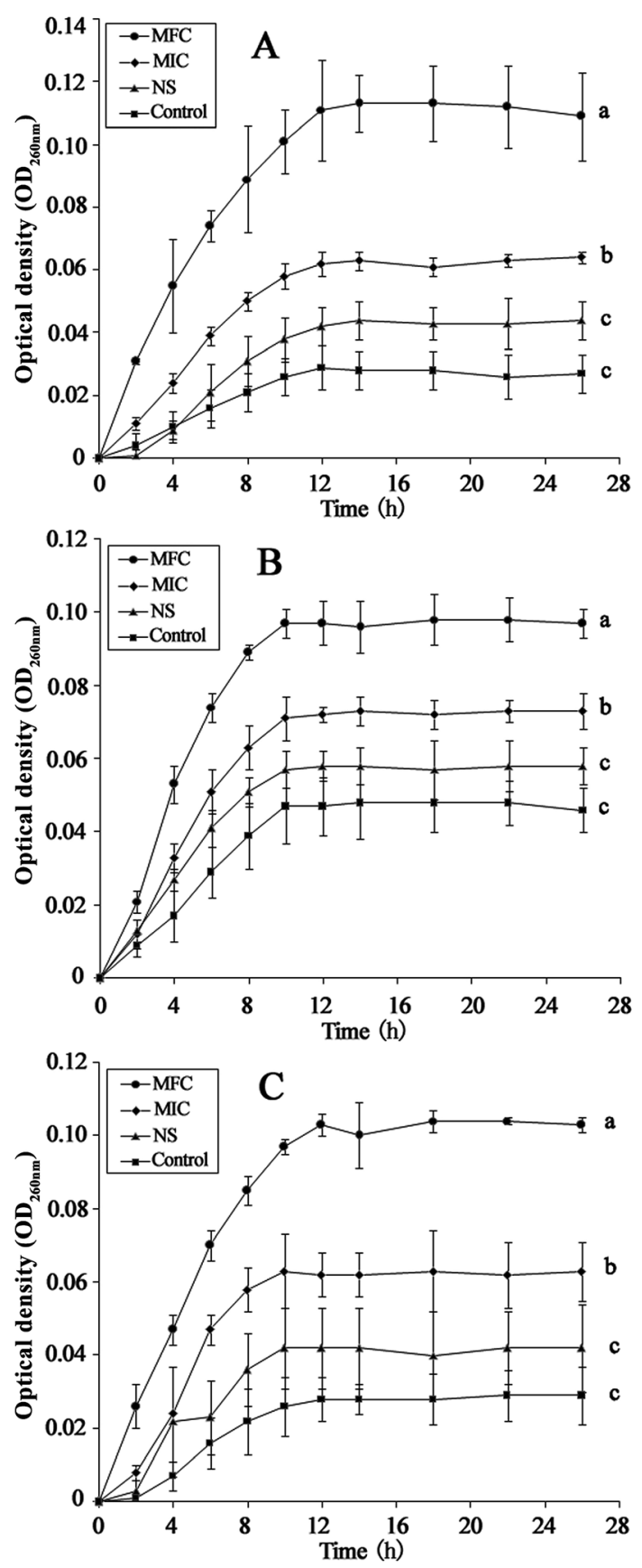

Fig. 3 Release of $260 \mathrm{~nm}$ absorbing material from E. floccosum (A), T. mentagrophytes (B), and T. rubrum (C) treated with NS and HTS. Minimum fungicidal concentration (MFC) of heat-transformed saponins; minimum inhibitory concentration (MIC) of heat-transformed saponins; NS, $0.125 \mathrm{mg} \mathrm{mL}^{-1}$ of raw notoginseng saponins; control, DMSO (6.4\%). Data (at $26 \mathrm{~h}$ ) followed by different letters at the end of each curve are significantly different $(P<0.05)$. dermatophytes were significantly increased $(p<0.05)$, compared with those of the control at $28 \mathrm{~h}$. The absorbance value for nucleic acids $\left(\mathrm{OD}_{260 \mathrm{~nm}}\right)$ of $E$. floccosum increased from $0 \mathrm{~h}$ to $12 \mathrm{~h}$. After $12 \mathrm{~h}$, the $\mathrm{OD}_{260 \mathrm{~nm}}$ value of E. floccosum showed almost no growth. Under similar conditions, there was
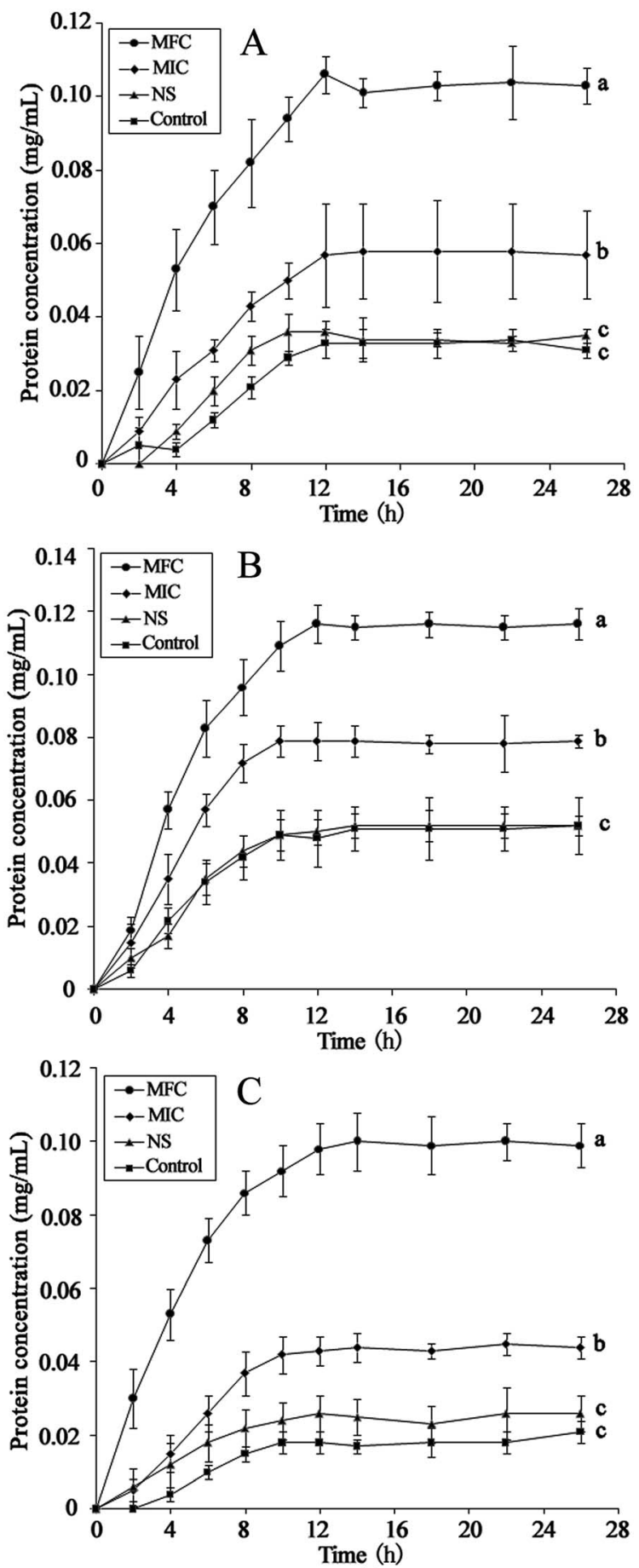

Fig. 4 Release of protein from E. floccosum (A), T. mentagrophytes (B), and T. rubrum (C) treated with NS and HTS. MFC of heat-transformed saponins; MIC of heat-transformed saponins; NS, $0.125 \mathrm{mg}$ $\mathrm{mL}^{-1}$ of raw notoginseng saponins; control, DMSO (6.4\%). Data (at 26 h) followed by different letters at the end of each curve are significantly different $(P<0.05)$. 


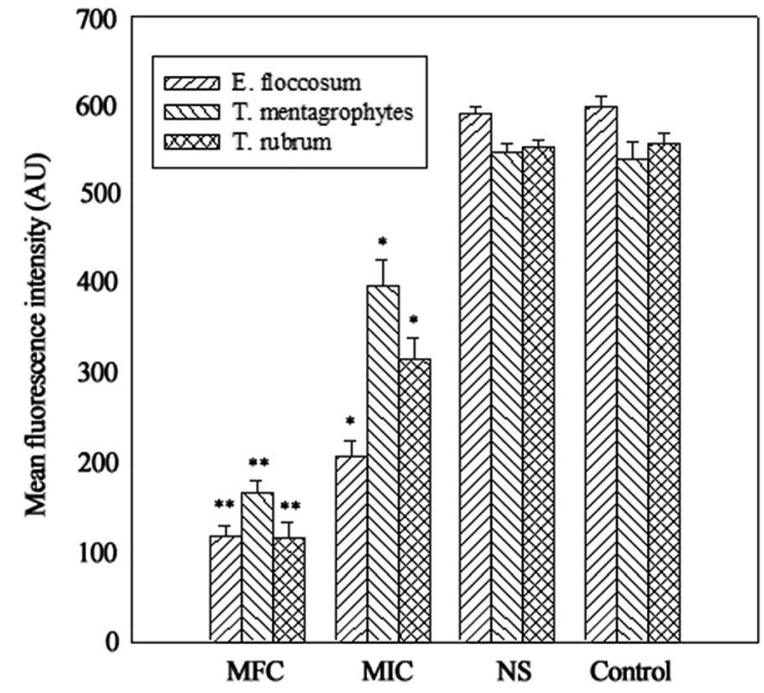

Fig. 5 Membrane potential (MP) of E. floccosum, T. mentagrophytes, and T. rubrum treated with HTS. MFC of heat-transformed saponins: MIC of heat-transformed saponins; NS, $0.5 \mathrm{mg} \mathrm{mL}^{-1}$ of raw notoginseng saponins; control, phosphate-buffered saline. * and ** mean $P$ $<0.05$ and $<0.01$, respectively.

a progressive release of nucleic acids of T. mentagrophytes and $T$. rubrum for up to almost 12 , $\mathrm{h}$ followed by a steady state. In addition, at $26 \mathrm{~h}$, the released protein concentrations of all the strains also increased significantly $(p<0.05)$ after the addition of HTS, particularly at the concentration of MFC. Interestingly, fungi treated with NS showed no significant difference from the control on $\mathrm{OD}_{260} \mathrm{~nm}$ values and protein concentrations. This result explicitly indicates that fungal cell membrane integrity was compromised after exposure to less polar ginsenosides.

The membrane potential of normal fungus was generated by the difference in the concentration of ions on opposite sides of the cell membrane. MP alteration of fungus could affect its cell metabolic activity. ${ }^{41}$ In this study, MP was illuminated by MFI of rhodamine 123, and the result is presented in Fig. 5. After the addition of HTS at the concentration of MIC, the MFI value of fungi was reduced by $65.33,26.44$ and 42.71 percent compared with the control, respectively. Furthermore, HTS at the concentration of MFC induced 80.01, 69.31, and 79.21 percent decrease of MFI for the fungi, respectively. However, NS did not significantly reduce the MFI of fungi. In the present study, fungal MP was directly correlated with the MFI. Therefore, HTS could significantly reduce the MP of dermatophytes. The reduction of MP revealed that cell membrane depolarization leads to irregular cell metabolic activity and fungus death.

\section{Conclusion}

This study clearly indicated that HTS exerted higher antifungal activities than those of NS. One major reason that explained this fact was that less polar ginsenosides in HTS were easier to interact with the fungal cell membranes and damage the integrity of the membrane, as well as decreasing the membrane potential. Based on the structure-activity relationships and wide spectrum against dermatophytes, HTS from notoginseng could be applied as antifungal agents to treat ringworm.

\section{Acknowledgements}

This study was supported by the International Science and Technology Cooperation Program of China (2015DFA31290) and the Agricultural Science and Technology Program for Innovation Team on Minor Grain Nutrition and Function, CAAS.

\section{References}

1 M. L. Santos, C. F. Maqlhaes, M. B. da Rosa, D. de Assis Santos, B. G. Brasileiro, L. M. de Carvalho, M. B. da Silva, C. L. Zani, E. P. de Siqueira, R. L. Peres and A. A. Andrade, Antifungal activity of extracts from Piper aduncum leaves prepared by different solvents and extraction techniques against dermatophytes Trichophyton rubrum and Trichophyton interdigitale, Braz. J. Microbiol., 2014, 4, 1275-1278.

2 N. T. Peres, F. C. Maranhao, A. Rossi and N. M. Martinez-Rossi, Dermatófitos: interação patógeno-hospedeiro e resistência a antifúngicos, An. Bras. Dermatol., 2010, 85, 657-667.

3 C. Seebacher, J. P. Bouchara and B. Mignon, Updates on the epidemiology of dematophyte infection, Mycopathologia, 2008, 166, 335-352.

4 S. Bassiri-Jahromi and A. A. Khaksari, Epidemiological survey of dermatophytosis in Tehran, Iran, from 2000 to 2005, Indian J. Dermatol. Venereol., 2009, 75, 142-147.

5 H. C. Gugnani, L. E. Akpata, M. K. Gugnani, et al., Isoconazole nitrate in the treatment of tropical dermatomycoses, Mycoses, 1994, 37, 39-41.

6 C. Romagnoli, D. Mares, A. Bruni, et al., Antifungal activity of 5 new synthetic compounds vs. Trichophyton rubrum and Epidermophyton floccosum, Mycopathologia, 2002, 153, 129-132.

7 D. Chattopadhyay, K. Maiti, A. P. Kundu, et al., Antimicrobial activity of Alstonia macrophylla: a folklore of bay islands, J. Ethnopharmacol., 2001, 77, 49-55.

8 M. R. Sartori, J. B. Pretto, A. B. Cruz, et al., Antifungal activity of fractions and two pure compounds of flowers from Wedelia paludosa (Acmela brasiliensis) (Asteraceae), Pharmazie, 2003, 58, 567-569.

9 Y. Tamura, K. Mizutani, T. Ikeda, et al., Antimicrobial activities of saponins of pericarps of Sapindus mukurossi on dermatophytes, Nat. Med., 2001, 55, 11-16.

10 J. D. Cleary, K. R. Stover, J. Farley, et al., Cardiac Toxicity of Azole Antifungals, Pharmacol. Pharm., 2013, 3, 362-368.

11 T. Heise, S. Rieke, F. Schmidt, et al., Analysis of hepatotoxic mixture effects of (tri)azole fungicides in a broad dose range, Toxicol. Lett., 2013, 221, S197.

12 I. Benko, F. Herndi, A. Megyeri, et al., Comparison of the toxicity of fluconazole and other azole antifungal drugs to murine and, human granulocyte, -macrophage progenitor, cells in vitro, J. Antimicrob. Chemother., 1999, 43, 675-681.

13 D. J. Conklin and P. J. Boor, Allylamine cardiovascular toxicity: evidence for aberrant vasoreactivity in rats, Toxicol. Appl. Pharmacol., 1998, 148, 245-251. 
14 P. J. Boor and R. M. Hysmith, Allylamine cardiovascular toxicity, Toxicology, 1987, 44, 129-145.

15 K. Ramos, S. L. Grossman and L. R. Cox, Allylamine-induced vascular toxicity in vitro: prevention by semicarbazidesensitive amine oxidase inhibitors, Toxicol. Appl. Pharmacol., 1988, 95, 61-71.

16 M. Mahboubi and N. Kazempour, The antifungal activity of Artemisia sieberi essential oil from different localities of Iran against dermatophyte fungi, J. Mycol. Med., 2015, 25, e65-e67.

17 M. Zuzarte, M. J. Goncalves, C. Cavalerio, M. T. Cruz, A. Benzarti, B. Marongiu, A. Maxia, L. Piras and L. Salgueiro, Antifungal and anti-inflammatory potential of Lavandula stoechas and Thymus herba-barona essential oils, Ind. Crops Prod., 2013, 44, 97-103.

18 J. Tian, X. B. Zeng, S. Zhang, Y. Z. Wang, P. Zhang, A. J. Lu and $\mathrm{X}$. Peng, Regional variation in components and antioxidant and antifungal activities of Perilla frutescens essential oils in China, Ind. Crops Prod., 2013, 59, 69-79.

19 S. Sun, C. Z. Wang, R. Tong, X. L. Li, A. Fishbein and Q. Wang, Effects of steaming the root of Panax notoginseng on chemical composition and anticancer activities, Food Chem., 2010, 118, 307-314.

20 T. B. Ng, Pharmacological activity of Sanchi ginseng (panax notognseng), J. Pharm. Pharmacol., 2006, 8, 1007-1019.

21 E. Barile, G. Bonanomi, V. Antignani, et al., Saponins from Allium minutiflorum, with antifungal activity, Phytochemistry, 2007, 68, 596-603.

22 Z. Du, N. Zhu, Z. Ren, et al., Two new antifungal saponins from the Tibetan herbal medicine Clematis tangutica, Planta Med., 2003, 69, 547-551.

23 M. Shimoyamada, M. Suzuki, H. Sonta, et al., Antifungal Activity of the Saponin Fraction Obtained from L. and Its Active Principle, Agric. Biol. Chem., 1990, 54, 2553-2557.

24 M. Sautour, T. Miyamoto and M. A. Lacailledubois, Steroidal saponins from Smilax medica and their antifungal activity, $J$. Nat. Prod., 2005, 68, 1489-1493.

25 S. W. Kwon, B. H. Sang and I. H. Park, Liquid chromatographic determination of less polar ginsenosides in processed ginseng, J. Chromatogr. A., 2001, 921, 335-339.

26 K. Quan, Q. Liu, J. Y. Wan, Y. J. Zhao, R. Z. Guo, R. N. Alolga, P. Li and L. W. Qi, Rapid preparation of rare ginsenosides by acid transformation and their structure-activity relationships against cancer cells, Sci. Rep., 2015, DOI: 10.1038/srep08598.

27 B. S. Sun, M. Y. Xu, Z. Li, Y. B. Wang and C. K. Sung, UPLC-QTOF-MS/MS analysis for steaming times-dependent profiling of steamed Panax quinquefolius and its ginsenosides transformations induced by repetitious steaming, $J$. Ginseng Res., 2012, 36, 277-290.

28 S. Sun, L. W. Qi, G. J. Du, S. Mehendale, C. Z. Wang and C. S. Yuan, Red notoginseng: higher ginsenoside content and stronger anticancer potential than Asian and American ginseng, Food Chem., 2011, 125, 1299-1305.

29 P. Xue, Y. Yao, X. S. Yang, J. Feng and G. X. Ren, Improved antimicrobial effect of ginseng extract by heat transformation, J. Ginseng Res., 2016, DOI: 10.1016/j.jgr.2016.03.002.

$30 \mathrm{X}$. P. Yang and X. D. Jiang, Antifungal activity and mechanism of tea polyphenols against Rhizopus stolonifer, Biotechnol. Lett., 2015, 37, 1463-1472.

31 I. Kubo and M. Himejima, Potentiation of antifungal activity of sesquiterpene dialdehydes against Candida albicans and two other fungi, Cell. Mol. Life Sci., 1992, 48, 1162-1164.

32 Y. B. Zhang, X. Y. Liu, Y. F. Wang, P. P. Jiang and S. Y. Quek, Antibacterial activity and mechanism of cinnamon essential oil against Escherichia coli and Staphylococcus aureus, Food Control, 2015, 59, 282-289.

$33 \mathrm{M}$. Bradford, A rapid and sensitive method for the quantitation of microgram quantities of protein utilizing the principle of protein-dye binding, Anal. Biochem., 1976, 72, 248-254.

$34 \mathrm{~J}$. Comas and J. Vives-Rego, Assessment of the effects of gramicidin, formaldehyde, and surfactants on Escherichia coli by flow cytometry using nucleic acid and membrane potential dyes, Cytometry, 1997, 29, 58-64.

35 Y. C. Zhang, Z. F. Pi, C. M. Liu, F. R. Song, Z. Q. Liu and S. Y. Liu, Analysis of low-polar ginsenosides in steamed Panax ginseng at high-temperature by HPLC-ESI-MS/MS, Chem. Res. Chin. Univ., 2012, 28, 31-36.

36 Y. Y. Xie, D. Luo, Y. J. Chen, J. F. Ma, Y. M. Wang, Q. L. Liang and G. A. Luo, Steaming-induced chemical transformations and holistic quality assessment of red ginseng derived from by means of HPLC-ESI-MS/MS ${ }^{n}$-based multicomponent quantification fingerprint, J. Agric. Food Chem., 2012, 60, 8213-8224.

37 H. Wu, B. L. Lee, L. Yang and H. G. Wang, Effects of ginseng on Pseudomonas aeruginosa motility and biofilm formation, FEMS Immunol. Med. Microbiol., 2011, 62, 49-56.

38 E. A. Bae, M. J. Han, M. K. Choo, S. Y. Park and D. H. Kim, Metabolism of $20(S)$ - and $20(R)$-ginsenoside $\mathrm{Rg}_{3}$ by human intestinal bacteria and its relation to in vitro biological activities, Biol. Pharm. Bull., 2002, 25, 58-63.

39 L. J. Wang, X. Yang, X. S. Yu, Y. Yao and G. X. Ren, Evaluation of antibacterial and anti-inflammatory activities of less polar ginsenosides produced from polar ginsenosides by heattransformation, J. Agric. Food Chem., 2013, 61, 12274-12282.

40 V. K. Bajpai, A. Sharma and K. H. Baek, Antibacterial mode of action of Cudrania tricuspidata fruit essential oil, affecting membrane permeability and surface characteristics of foodborne pathogens, Food Control, 2013, 32, 582-590.

41 C. Spampinato and D. Leonardi, Candida infections, causes, targets and resistance mechanisms: traditional and alternative antifungal agents, BioMed Res. Int., 2013, 2013, 204-237. 